STONE DISEASE

doi: 10.1590/S1677-553820100003000014

\title{
Chronic kidney disease affects the stone-free rate after extracorporeal shock wave lithotripsy for proximal ureteric stones
}

Hung SF, Chung SD, Wang SM, Yu HJ, Huang HS

Department of Urology, National Taiwan University Hospital and College of Medicine, National Taiwan University, Taipei, Taiwan

BJU Int. 2009 Nov 20. [Epub ahead of print]

Objective: To investigate the effect of renal function on the stone-free rate (SFR) of proximal ureteric stones (PUS) after extracorporeal shock wave lithotripsy (ESWL), as urinary obstruction caused by PUS can impair renal function, and elevated serum creatinine levels are associated with decreased ureteric stone passage.

Patients And Methods: From January 2005 to December 2007, 1534 patients had ESWL for urolithiasis, 319 having ESWL in situ for PUS; they were reviewed retrospectively. Patients requiring simultaneous treatment of kidney stones, placement of a double pigtail stent, or percutaneous pigtail nephrostomy tube were excluded. We divided patients into groups by chronic kidney disease (CKD) stage according to the estimated glomerular filtration rate (eGFR) of $>/=60$ and $<60 \mathrm{~mL} / \mathrm{min} / 1.73 \mathrm{~m}(2)$. Stone-free status was defined as no visible stone fragments on a plain abdominal film at 3 months after ESWL. A logistic regression model was used to evaluate the possible significant factors that influenced the SFR of PUS after ESWL, and to develop a prediction model.

Results: The overall SFR of PUS (276/319 patients) was $86.5 \%$; the SFR was $93 \%$ in patients with an eGFR of $>1=60$ and $50 \%$ in those with an eGFR of $<60(\mathrm{P}<0.001)$. After univariate and multivariate analysis, the three significant factors affecting SFR were an eGFR of $>/=60$, stone width, and gender, with odds ratios $(95 \%$ confidence intervals) of $19.54(8.25-46.30)(\mathrm{P}<0.001), 0.67(0.55-0.82)(\mathrm{P}<0.001)$ and $0.16(0.05-0.50(\mathrm{P}=$ 0.002), respectively. A logistic regression model was developed to estimate the probability of SFR after ESWL, the equation being $1 /(1+\exp [-(3.8137-0.3967 \times$ (stone width $)+2.9724 \times$ eGFR $-1.8120 \times$ Male $)])$, where stone width is the observed value (mm), eGFR $=1$ for eGFR $>/=60$ and 0 for $<60$, and male $=1$ for male, 0 for female.

Conclusions: Gender, eGFR $>/=60$ and a stone width of $>7 \mathrm{~mm}$ were significant predictors affecting the SFR after one session of ESWL for PUS.

\section{Editorial Comment}

The authors do not state at what time point was the serum creatinine obtained that was utilized to calculate the estimated GFR. This is a critical omission. Ideally the serum $\mathrm{Cr}$ and GFR would have been evaluated after resolution of the obstructing calculus. This would identify those with true chronic kidney disease. In contrast, if these values were evaluated at the time of obstruction; the abnormality may have been post-renal. Indeed, if they have selected those patients with renal insufficiency due to severe obstruction, one would anticipate that these may be patients with more severe hydronephrosis or longer duration since onset of pain and obstruction; both of which could be independent predictors of failure of SWL. The observation that stone width is more critical than stone length in determining shockwave success may be important to consider when counseling patients.

\author{
Dr. Manoj Monga \\ Department of Urology \\ Cleveland Clinic Foundation \\ Cleveland, Ohio, USA \\ E-mail:endourol@yahoo.com
}

